\title{
Almost Kenmotsu 3-h-metric as a cotton soliton
}

\author{
Dibakar Dey and Pradip Majhi \\ Department of Pure Mathematics, University of Calcutta, Kolkata, India
}

\begin{abstract}
Purpose - Cotton soliton is a newly introduced notion in the field of Riemannian manifolds. The object of this article is to study the properties of this soliton on certain contact metric manifolds.

Design/methodology/approach - The authors consider the notion of Cotton soliton on almost Kenmotsu 3-manifolds. The authors use a local basis of the manifold that helps to study this notion in terms of partial differential equations.

Findings - First the authors consider that the potential vector field is pointwise collinear with the Reeb vector field and prove a non-existence of such Cotton soliton. Next the authors assume that the potential vector field is orthogonal to the Reeb vector field. It is proved that such a Cotton soliton on a non-Kenmotsu almost Kenmotsu 3-h-manifold such that the Reeb vector field is an eigen vector of the Ricci operator is steady and the manifold is locally isometric to.

Originality/value - The results of this paper are new and interesting. Also, the Proposition 3.2 will be helpful in further study of this space.
\end{abstract}

Keywords Almost Kenmotsu manifolds, Cotton solitons, Non-unimodular lie group, Product space Paper type Research paper

\section{Introduction}

An almost contact metric manifold is an odd dimensional differentiable manifold $M^{2 n+1}$ together with a structure $(\varphi, \xi, \eta, g)$ satisfying $([1,2])$

$$
\begin{gathered}
\varphi^{2} X=-X+\eta(X) \xi, \quad \eta(\xi)=1, \\
g(\varphi X, \varphi Y)=g(X, Y)-\eta(X) \eta(Y)
\end{gathered}
$$

for all vector fields $X$, $Y$ on $M^{2 n+1}$, where $g$ is the Riemannian metric, $\varphi$ is a (1,1)-tensor field, $\xi$ is a unit vector field called the Reeb vector field and $\eta$ is a 1-form defined by $\eta(X)=g(X, \xi)$. Here also $\phi \xi=0$ and $\eta \circ \phi=0$; both can be derived from (1.1) easily. The fundamental 2 -form $\Phi$ on an almost contact metric manifold is defined by $\Phi(X, Y)=g(X, \varphi Y)$ for all vector fields $X$, $Y$ on $M^{2 n+1}$. The condition for an almost contact metric manifold being normal is equivalent to vanishing of the (1,2)-type torsion tensor $N_{\varphi}$, defined by $N_{\varphi}=[\varphi, \varphi]+2 d \eta \otimes \xi$, where $[\varphi, \varphi]$ is the Nijenhuis tensor of $\varphi$ [1]. An almost contact metric manifold such that $\eta$ is closed and $d \Phi=2 \eta \wedge \Phi$ is called almost Kenmotsu manifold (see [3, 4]). Obviously, a normal almost Kenmotsu manifold is a Kenmotsu manifold. Also Kenmotsu manifolds can be characterized

\section{JEL Classification — Primary 53D15; Secondary 35Q51.}

(C) Dibakar Dey and Pradip Majhi. Published in the Arab Journal of Mathematical Sciences. Published by Emerald Publishing Limited. This article is published under the Creative Commons Attribution (CC BY 4.0) license. Anyone may reproduce, distribute, translate and create derivative works of this article (for both commercial and non-commercial purposes), subject to full attribution to the original publication and authors. The full terms of this license may be seen at http://creativecommons.org/licences/by/4.0/ legalcode

The authors gratefully acknowledge the valuable comments of the anonymous referees and express their sincere thanks to them. The preprint version of this article is available in https://arxiv.org/abs/ 2006.122 44. The author Dibakar Dey is thankful to the Council of Scientific and Industrial Research, India (File no: 09/028(1010)/2017-EMR-1) for their assistance in the form of Senior Research Fellowship.

Received 30 October 2020 Revised 27 February 2021 16 May 2021

Accepted 1 January 2022 
by $\left(\nabla_{X} \varphi\right) Y=g(\varphi X, Y) \xi-\eta(Y) \varphi X$, for any vector fields $X, Y$ on $M^{2 n+1}$. For further details on Kenmotsu manifolds we refer the reader to go through the references $([5,6])$.

The Weyl tensor on an $n$-dimensional Riemannian manifold is defined as.

$$
\begin{aligned}
\mathcal{C}(X, Y) Z= & R(X, Y) Z+\frac{r}{(n-1)(n-2)}[g(Y, Z) X-g(X, Z) Y] \\
& -\frac{1}{n-2}[S(Y, Z) X-S(X, Z) Y+g(Y, Z) Q X-g(X, Z) Q Y]
\end{aligned}
$$

where $R$ is the curvature tensor, $S$ denotes the Ricci tensor, $Q$ stands for Ricci operator and $r$ is the scalar curvature.

A $(0,3)$-Cotton tensor of a 3-dimensional Riemannian manifold $\left(M^{3}, g\right)$ is defined as (see [7])

$$
\begin{aligned}
C(X, Y, Z)= & \left(\nabla_{X} S\right)(Y, Z)-\left(\nabla_{Y} S\right)(X, Z) \\
& -\frac{1}{4}[X(r) g(Y, Z)-Y(r) g(X, Z)],
\end{aligned}
$$

where $S$ is the Ricci tensor and $r$ is the scalar curvature of $M^{3}$. The Cotton tensor is skew-symmetric in first two indices and totally trace free. It is well known that for $n \geq 4$, an $n$-dimensional Riemannian manifold is conformally flat if the Weyl tensor vanishes. For $n=3$, the Weyl tensor always vanishes but the Cotton tensor does not vanish in general.

In 2008, Kicisel, Sarioglu and Tekin [8] introduced the notion of Cotton flow as an analogy of the Ricci flow. The Cotton flow is based on the conformally invariant Cotton tensor and defined exclusively for 3-dimension as

$$
\frac{\partial g}{\partial t}=C
$$

where $C$ is the $(0,2)$-Cotton tensor of $g$. From the Cotton flow, they defined the notion of Cotton soliton as follows:

Definition 1.1. A Cotton soliton is a metric g defined on 3-dimensional smooth manifold $M^{3}$ such that the following equation

$$
\left(\mathcal{L}_{V} g\right)(X, Y)+C(X, Y)-\sigma g(X, Y)=0,
$$

holds for a constant $\sigma$ and a vector field $V$, called the potential vector field, where $\mathcal{L}_{V}$ denotes the Lie derivative along $V$ and $C$ is the $(0,2)$-Cotton tensor defined by

$$
C_{i j}=\frac{1}{2 \sqrt{g}} C_{n m i} \epsilon^{n m l} g_{l j}
$$

in a local frame of $M^{3}$, where $g=\operatorname{det}\left(g_{i j}\right), C_{i j k}$ is the $(0,3)$-Cotton tensor and $\epsilon$ is a tensor density. In an orthonormal frame, $\epsilon^{123}=1$. Also exchange of any two indices will give rise to minus sign and it will be zero if there has two same indices. For example, $\epsilon^{231}=-\epsilon^{213}$ and $\epsilon^{112}=\epsilon^{122}=\epsilon^{223}=0$. Cotton solitons are fixed points of the Cotton flow up to diffeomorphisms and rescaling. The Cotton soliton is said to be shrinking, steady or expanding according as $\sigma$ is positive, zero or negative respectively. As far as we know, the Cotton soliton was studied by Chen [9] on certain almost contact metric manifold, precisely on almost coKähler 3-manifolds. Motivated by the study of Chen [9], we consider the notion of Cotton soliton on an almost Kenmotsu 3 - $h$-manifold and prove some related results. 
2. Almost Kenmotsu 3- $\boldsymbol{h}$-manifolds

Let $\left(M^{3}, \varphi, \xi, \eta, g\right)$ be a 3-dimensional almost Kenmotsu manifold. We denote by $l=R(\cdot, \xi) \xi$, $h=\frac{1}{2} \mathcal{L}_{\xi} \varphi$ and $h^{\prime}=h \circ \varphi$ on $M^{3}$, where $R$ is the Riemannian curvature tensor. The tensor fields $l$ and $h$ are symmetric operators and satisfy the following relations ([3, 4]):

$$
\begin{gathered}
h \xi=0, l \xi=0, \operatorname{tr}(h)=0, \operatorname{tr}(h \varphi)=0, h \varphi+\varphi h=0, \\
\nabla_{X} \xi=X-\eta(X) \xi-\varphi h X\left(\Rightarrow \nabla_{\xi} \xi=0\right), \\
\nabla_{\xi} h=-\varphi-2 h-\varphi h^{2}-\varphi l .
\end{gathered}
$$

Definition 2.1. [10] A 3-dimensional almost Kenmotsu manifold is called an almost Kenmotsu 3-h-manifold if it satisfies $\nabla_{\xi} h=0$.

Let $\mathcal{U}_{1}$ be the maximal open subset of a 3-dimensional almost Kenmotsu manifold $M^{3}$ such that $h \neq 0$ and $\mathcal{U}_{2}$ be the maximal open subset on which $h=0$. Then $\mathcal{U}_{1} \mathcal{U}_{2}$ is an open and dense subset of $M^{3}$. Then $\mathcal{U}_{1}$ is non-empty and there is a local orthonormal basis $\left\{e_{1}=\xi\right.$, $\left.e_{2}=e, e_{3}=\varphi e\right\}$ on $\mathcal{U}_{1}$ such that $h e=\lambda e$ and $h \varphi e=-\lambda \varphi e$ for some positive function $\lambda$.

Lemma 2.2. [11] $\mathrm{OnU}_{1}$,

$$
\begin{gathered}
\nabla_{\xi} \xi=0, \quad \nabla_{\xi} e=a \varphi e, \quad \nabla_{\xi} \varphi e=-a e \\
\nabla_{e} \xi=e-\lambda \varphi e, \quad \nabla_{e} e=-\xi-b \varphi e, \quad \nabla_{e} \varphi e=\lambda \xi+b e \\
\nabla_{\varphi e} \xi=-\lambda e+\varphi e, \quad \nabla_{\varphi e} e=\lambda \xi+c \varphi e, \quad \nabla_{\varphi e} \varphi e=-\xi-c e
\end{gathered}
$$

where $a, b$ and $c$ are smooth functions.

Since $\nabla_{\xi} h=0$ for an almost Kenmotsu 3- $h$-manifold, then using Lemma 2.2 and (2.3), a direct calculation gives $\xi(\lambda)=a=0$. Therefore, Lemma 2.2 can be rewritten for an almost Kenmotsu 3 - $h$-manifold as.

Lemma 2.3. On $\mathcal{U}_{1}$, the coefficients of the Riemannian connection $\nabla$ of an almost Kenmotsu 3-h-manifold with respect to a local orthonormal basis $\{\xi, e, \varphi e\}$ is given by

$$
\begin{gathered}
\nabla_{\xi} \xi=0, \quad \nabla_{\xi} e=0, \quad \nabla_{\xi} \phi e=0, \\
\nabla_{e} \xi=e-\lambda \varphi e, \quad \nabla_{e} e=-\xi-b \varphi e, \quad \nabla_{e} \varphi e=\lambda \xi+b e \\
\nabla_{\varphi e} \xi=-\lambda e+\varphi e, \quad \nabla_{\varphi e} e=\lambda \xi+c \varphi e, \quad \nabla_{\varphi e} \varphi e=-\xi-c e,
\end{gathered}
$$

where $b$ and $c$ are smooth functions.

From Lemma 2.3, the Lie brackets can be calculated as follows:

$$
[e, \xi]=e-\lambda \varphi e, \quad[e, \varphi e]=b e-c \varphi e \text { and }[\varphi e, \xi]=-\lambda e+\varphi e .
$$

In [12], Wang obtained the components of the Ricci operator $Q$ for an almost Kenmotsu 3manifold on $\mathcal{U}_{1}$ as follows:

$$
\begin{gathered}
Q \xi=-2\left(\lambda^{2}+1\right) \xi-\sigma(e) e-\sigma(\varphi e) \varphi e \\
Q e=-\sigma(e) \xi-(f+2 \lambda a) e+(\xi(\lambda)+2 \lambda) \varphi e \\
Q \varphi e=-\sigma(\varphi e) \xi+(\xi(\lambda)+2 \lambda) e-(f-2 \lambda a) \varphi e
\end{gathered}
$$


where $f=e(c)+\varphi e(b)+b^{2}+c^{2}+2$ and $\sigma(\cdot)=-g(Q \xi$, $\cdot)$. Now, we write the components of the Ricci operator $Q$ for an almost Kenmotsu 3- $h$-manifold as follows:

Lemma 2.4. $O n \mathcal{U}_{1}$, the Ricci operator of an almost Kenmotsu 3-h-manifold with respect to a local orthonormal basis $\{\xi, e, \varphi e\}$ is given by

$$
\begin{gathered}
Q \xi=-2\left(\lambda^{2}+1\right) \xi-[\varphi e(\lambda)+2 \lambda b] e-[e(\lambda)+2 \lambda c] \varphi e, \\
Q e=-[\varphi e(\lambda)+2 \lambda b] \xi-f e+2 \lambda \varphi e, \\
Q \varphi e=-[e(\lambda)+2 \lambda c] \xi+2 \lambda e-f \varphi e,
\end{gathered}
$$

where $f=e(c)+\varphi e(b)+b^{2}+c^{2}+2$.

The scalar curvature $r$ of an almost Kenmotsu 3-h-manifold is given by

$$
r=g\left(Q e_{i}, e_{i}\right)=-2\left(\lambda^{2}+1\right)-2 f .
$$

Using Lemma 2.4, we obtain

$$
\left\{\begin{array}{l}
S(\xi, \xi)=-2\left(\lambda^{2}+1\right), S(\xi, e)=-[\varphi e(\lambda)+2 \lambda b] \\
S(\xi, \varphi e)=-[e(\lambda)+2 \lambda c], S(e, e)=-f \\
S(e, \varphi e)=2 \lambda, S(\varphi e, \varphi e)=-f
\end{array}\right.
$$

It is well known that an almost Kenmotsu 3-manifold is Kenmotsu if and only if $h=0$. Thus a Kenmotsu metric always admits an almost Kenmotsu 3 - $h$-metric structure. We now close this section by providing an example of a non-Kenmotsu almost Kenmotsu 3- $h$-manifold.

Example 2.5. [13] Let $M^{3}$ be a 3-dimensional non-unimodular Lie group with a left invariant local orthonormal frame $\left\{e_{1}, e_{2}, e_{3}\right\}$ satisfying

$$
\left[e_{1}, e_{2}\right]=\alpha e_{2}+\beta e_{3},\left[e_{2}, e_{3}\right]=0 \text { and }\left[e_{1}, e_{3}\right]=\beta e_{2}+(2-\alpha) e_{3}
$$

for $\alpha, \beta \in \mathbb{R}$ If either $\alpha \neq 1$ or $\beta \neq 0$, then $M^{3}$ admits a non-Kenmotsu almost Kenmotsu 3-h-metric structure.

We now close this section by recalling an important result of Cho [14].

Theorem 2.6. A non-Kenmotsu almost Kenmotsu 3-manifold $M^{3}$ is locally symmetric if and only if $M^{3}$ is locally isometric to the product space $\mathbb{H}^{2}(-4) \times \mathbb{R}$

\section{Cotton soliton}

In this section, we consider the notion of Cotton soliton within the framework of almost Kenmotsu 3-h-manifolds. To study the notion of Cotton soliton, we need to compute the components of the $(0,2)$-Cotton tensor. In this regard, we prove the following Lemma:

Lemma 3.1. The components of the $(0,2)$-Cotton tensor $C$ with respect to an orthonormal frame $\{\xi, e, \varphi$ e $\}$ of a non-Kenmotsu almost Kenmotsu 3-h-manifold $M^{3}$ can be expressed as follows:

$$
\begin{aligned}
C_{11}=C(\xi, \xi)= & b[\varphi e(\lambda)+2 \lambda b]-c[e(\lambda)+2 \lambda c] \\
& -e(e(\lambda)+2 \lambda c)+\varphi e(\varphi e(\lambda)+2 \lambda b),
\end{aligned}
$$




$$
\begin{aligned}
& C_{12}=C(\xi, e)=2\left[e(\lambda)-3 \lambda \varphi e(\lambda)+2 \lambda c-2 \lambda^{2} b\right] \\
& +\xi(e(\lambda)+2 \lambda c)-\frac{1}{4} \varphi e(r), \\
& C_{13}=C(\xi, \varphi e)=-2\left[\varphi e(\lambda)-3 \lambda e(\lambda)+2 \lambda b-2 \lambda^{2} c\right] \\
& -\xi(\varphi e(\lambda)+2 \lambda b)+\frac{1}{4} e(r), \\
& C_{22}=C(e, e)=2 \lambda^{3}-f \lambda+c[e(\lambda)+2 \lambda c]-\varphi e(\varphi e(\lambda)+2 \lambda b), \\
& C_{23}=C(e, \varphi e)=-\xi(f)-f+2+e(\varphi e(\lambda)+2 \lambda b)+b[e(\lambda)+2 \lambda c]-\frac{1}{4} \xi(r), \\
& C_{33}=C(\varphi e, \varphi e)=-2 \lambda^{3}+f \lambda-b[\varphi e(\lambda)+2 \lambda b]+e(e(\lambda)+2 \lambda c) .
\end{aligned}
$$

Proof. The components of the metric tensor $g$ with respect to an orthonormal frame $\{\xi, e, \varphi e\}$ of a non-Kenmotsu almost Kenmotsu 3- $h$-manifold $M^{3}$ is given by

$$
\left(g_{i j}\right)=\left(\begin{array}{lll}
1 & 0 & 0 \\
0 & 1 & 0 \\
0 & 0 & 1
\end{array}\right)
$$

and hence $\operatorname{det}\left(g_{i j}\right)=1$. Therefore, Eqn (1.5) reduces to

$$
C_{i j}=\frac{1}{2} C_{n m i} \epsilon^{n m j}, \quad i, j=1,2,3,
$$

where $C_{i j k}=C\left(e_{i}, e_{j}, e_{k}\right)$. Also, $C_{i j k}=-C_{j i k}$ and $C_{i i k}=0$ for all $i, j, k=1,2$, 3. It can be easily obtained that (see [9])

$$
C_{11}=C_{231}, C_{12}=C_{311}, C_{13}=C_{121}, C_{22}=C_{312}, C_{23}=C_{122}, C_{33}=C_{123} .
$$

Making use of (1.3), we get the following:

$$
\begin{gathered}
C_{11}=C_{231}=C(e, \varphi e, \xi)=\left(\nabla_{e} S\right)(\varphi e, \xi)-\left(\nabla_{\varphi e} S\right)(e, \xi), \\
C_{12}=C(\varphi e, \xi, \xi)=\left(\nabla_{\varphi e} S\right)(\xi, \xi)-\left(\nabla_{\xi} S\right)(\varphi e, \xi)-\frac{1}{4} \varphi e(r), \\
C_{13}=C(\xi, e, \xi)=\left(\nabla_{\xi} S\right)(e, \xi)-\left(\nabla_{e} S\right)(\xi, \xi)+\frac{1}{4} e(r), \\
C_{22}=C(\varphi e, \xi, e)=\left(\nabla_{\varphi e} S\right)(\xi, e)-\left(\nabla_{\xi} S\right)(\varphi e, e), \\
C_{23}=C(\xi, e, e)=\left(\nabla_{\xi} S\right)(e, e)-\left(\nabla_{e} S\right)(\xi, e)-\frac{1}{4} \xi(r), \\
C_{33}=C(\xi, e, \varphi e)=\left(\nabla_{\xi} S\right)(e, \varphi e)-\left(\nabla_{e} S\right)(\xi, \varphi e) .
\end{gathered}
$$

Using (2.6), Lemma 2.3 and $\xi(\lambda)=0$, we now obtain the following: 


$$
\begin{gathered}
\left\{\begin{array}{l}
\left(\nabla_{e} S\right)(\varphi e, \xi)=2 \lambda^{3}-f \lambda+b[\varphi e(\lambda)+2 \lambda b]-e(e(\lambda)+2 \lambda c), \\
\left(\nabla_{\varphi} S\right)(e, \xi)=2 \lambda^{3}-f \lambda+c[e(\lambda)+2 \lambda c]-\varphi e(\varphi e(\lambda)+2 \lambda b) .
\end{array}\right. \\
\left\{\begin{array}{l}
\left(\nabla_{\varphi e} S\right)(\xi, \xi)=2\left[e(\lambda)-3 \lambda \varphi e(\lambda)+2 \lambda c-2 \lambda^{2} b\right], \\
\left(\nabla_{\xi} S\right)(\varphi, \xi)=-\xi(e(\lambda)+2 \lambda c) .
\end{array}\right. \\
\left\{\begin{array}{l}
\left(\nabla_{\xi} S\right)(e, \xi)=-\xi(\varphi e(\lambda)+2 \lambda b), \\
\left(\nabla_{e} S\right)(\xi, \xi)=2\left[\varphi e(\lambda)-3 \lambda e(\lambda)+2 \lambda b-2 \lambda^{2} c\right] .
\end{array}\right. \\
\left\{\begin{array}{l}
\left(\nabla_{\varphi e} S\right)(\xi, e)=2 \lambda^{3}-f \lambda+c[e(\lambda)+2 \lambda c]-\varphi e(\varphi e(\lambda)+2 \lambda b), \\
\left(\nabla_{\xi} S\right)(\varphi e, e)=0 .
\end{array}\right. \\
\left\{\begin{array}{l}
\left(\nabla_{\xi} S\right)(e, e)=-\xi(f), \\
\left(\nabla_{e} S\right)(\xi, e)=f-2-e(\varphi e(\lambda)+2 \lambda b)-b[e(\lambda)+2 \lambda c] .
\end{array}\right. \\
\left\{\begin{array}{l}
\left(\nabla_{\xi} S\right)(e, \varphi e)=0, \\
\left(\nabla_{e} S\right)(\xi, \varphi e)=2 \lambda^{3}-f \lambda+b[\varphi e(\lambda)+2 \lambda b]-e(e(\lambda)+2 \lambda c) .
\end{array}\right.
\end{gathered}
$$

We now complete the proof by substituting Eqs (3.13)-(3.18) in Eqs (3.7)-(3.12) respectively.

Proposition 3.2. If the Reeb vector field of a non-Kenmotsu almost Kenmotsu 3-h-manifold $M^{3}$ is an eigen vector of the Ricci operator, then $M^{3}$ is locally isometric to a non-unimodular Lie group equipped with a left invariant non-Kenmotsu almost Kenmotsu structure.

Proof. Since $\xi$ is an eigen vector of $Q$, then Lemma 2.4 implies

$$
\left\{\begin{array}{l}
\varphi e(\lambda)+2 \lambda b=0 \\
e(\lambda)+2 \lambda c=0
\end{array}\right.
$$

It is well known that

$$
\frac{1}{2} X(r)=(\operatorname{div} Q) X=\sum_{i=1}^{3} g\left(\left(\nabla_{e_{i}} Q\right) X, e_{i}\right) .
$$

From the preceding equation, we can write

$$
\frac{1}{2} X(r)=\left(\nabla_{\xi} S\right)(X, \xi)+\left(\nabla_{e} S\right)(X, e)+\left(\nabla_{\varphi e} S\right)(X, \varphi e) .
$$

Making use of (2.6), (3.19) and $\xi(\lambda)=0$, we obtain the following:

$$
\begin{array}{r}
\left(\nabla_{\xi} S\right)(\xi, \xi)=0,\left(\nabla_{e} S\right)(\xi, e)=f-2,\left(\nabla_{\varphi e} S\right)(\xi, \varphi e)=f-2, \\
\left(\nabla_{\xi} S\right)(e, \xi)=0,\left(\nabla_{e} S\right)(e, e)=4 \lambda b-e(f),\left(\nabla_{\varphi e} S\right)(e, \varphi e)=-4 \lambda b, \\
\left(\nabla_{\xi} S\right)(\varphi e, \xi)=0,\left(\nabla_{e} S\right)(\varphi e, e)=-4 \lambda c,\left(\nabla_{\varphi e} S\right)(\varphi e, \varphi e)=4 \lambda c-\varphi e(f) .
\end{array}
$$

Now, substituting $X=\xi, e$ and $\varphi e$ in (3.20) and then using (3.21), (3.22) and (3.23) respectively, we obtain

$$
\xi(r)=4(f-2), \quad e(r)=-2 e(f), \quad \varphi e(r)=-2 \varphi e(f) .
$$


Using (3.19) and $\xi(\lambda)=0$, we get from (2.5)

$$
\xi(r)=-2 \xi(f), e(r)=-2 e(f)+8 \lambda^{2} c, \varphi e(r)=-2 \varphi e(f)+8 \lambda^{2} b .
$$

Since $\lambda$ is a positive function, then the second and third equations of (3.24) and (3.25) implies $b=c=0$. From Lemma 2.4, we get $f=2$. Also from (3.19), we get $e(\lambda)=\varphi e(\lambda)=0$ and therefore $\lambda$ is a constant. Now, the Lie brackets given in (2.4) reduces to

$$
[e, \xi]=e-\lambda \varphi e, \quad[e, \varphi e]=0 \text { and }[\varphi e, \xi]=-\lambda e+\varphi e
$$

Therefore, according to Milnor (Page 309, Lemma 4.10 [15]), $M^{3}$ is locally isometric to a nonunimodular Lie group equipped with a left invariant non-Kenmotsu almost Kenmotsu structure.

Combining Lemma 3.1 and Proposition 3.2, the components of the Cotton tensor described as:

Corollary 3.3. If the Reeb vector field of a non-Kenmotsu almost Kenmotsu 3-h-manifold $M^{3}$ is an eigen vector of the Ricci operator, then the components of the (0,2)-Cotton tensor $C$ with respect to an orthonormal frame $\{\xi, e, \varphi e\}$ on $M^{3}$ can be expressed as follows:

$$
\begin{gathered}
C_{11}=C(\xi, \xi)=0, C_{12}=C(\xi, e)=0, C_{13}=C(\xi, \varphi e)=0, \\
C_{22}=C(e, e)=2 \lambda^{3}-2 \lambda, C_{23}=C(e, \varphi e)=0, C_{33}=C(\varphi e, \varphi e)=-2 \lambda^{3}+2 \lambda .
\end{gathered}
$$

We first consider the Cotton soliton with potential vector field $V$ pointwise collinear with the Reeb vector field. In this regard, we prove the following non-existing result.

Theorem 3.4. On a non-Kenmotsu almost Kenmotsu 3-h-manifold such that the Reeb vector field is an eigen vector of the Ricci operator, there exist no Cotton soliton with potential vector field pointwise collinear with the Reeb vector field.

Proof. Suppose that the potential vector field $V$ is pointwise collinear with the Reeb vector field $\xi$. Then there exist a non-zero smooth function $\alpha$ on $M^{3}$ such that $V=\alpha \xi$. Now, substituting $X=e$ and $Y=\varphi e$ in (1.4) and using Lemma 2.3 and Corollary 3.3, we get $2 \lambda \alpha=0$. This gives either $\lambda=0$ or $\alpha=0$. In either cases, we get a contradiction. This completes the proof.

From Theorem 3.4 and Proposition 3.2, we have.

Corollary 3.5. On a 3-dimensional non-unimodular Lie group equipped with a left invariant non-Kenmotsu almost Kenmotsu structure, there exist no Cotton soliton with potential vector field pointwise collinear with the Reeb vector field.

It is now quite tempting to consider the potential vector field $V$ as orthogonal to the Reeb vector field. In this setting, we prove the following:

Theorem 3.6. Let $\left(M^{3}, g\right)$ be a non-Kenmotsu almost Kenmotsu 3-h-manifold such that the Reeb vector field is an eigen vector of the Ricci operator. If $g$ is a Cotton soliton with potential vector field orthogonal to the Reeb vector field, then $M^{3}$ is locally isometric to $\mathbb{U}^{2}(-4) \times \mathbb{R}$ and the Cotton soliton is steady.

Proof. For a non-Kenmotsu almost Kenmotsu 3- $h$-manifold such that the Reeb vector field is an eigen vector of the Ricci operator, Proposition 3.2 gives $b=c=0, f=2, \lambda=$ constant and $r=$ constant. Since $V$ is orthogonal to $\xi$, then there exist two smooth functions $\alpha_{1}$ and $\alpha_{2}$ on $M^{3}$ such that $V=\alpha_{1} e+\alpha_{2} \varphi e$. With the help of Lemma 2.3, we now obtain the components of $\mathcal{L}_{V} g$ as follows: 


$$
\left\{\begin{array}{l}
\left(\mathcal{L}_{V} g\right)(\xi, \xi)=0,\left(\mathcal{L}_{V} g\right)(\xi, e)=\xi\left(\alpha_{1}\right)-\alpha_{1}+\lambda \alpha_{2}, \\
\left(\mathcal{L}_{V} g\right)(\xi, \varphi e)=\xi\left(\alpha_{2}\right)-\alpha_{2}+\lambda \alpha_{1},\left(\mathcal{L}_{V} g\right)(e, e)=2 e\left(\alpha_{1}\right), \\
\left(\mathcal{L}_{V} g\right)(e, \varphi e)=e\left(\alpha_{2}\right)+\varphi e\left(\alpha_{1}\right),\left(\mathcal{L}_{V} g\right)(\varphi e, \varphi e)=2 \varphi e\left(\alpha_{2}\right) .
\end{array}\right.
$$

We now use Corollary 3.3 and (3.26). Substituting $X=Y=\xi$ in (1.4), we get $\sigma=0$. This shows that the Cotton soliton is steady. Now, substitution of $X=\xi, Y=e$ in (1.4) yields

$$
\xi\left(\alpha_{1}\right)-\alpha_{1}+\lambda \alpha_{2}=0 .
$$

Replacing $X$ by $\xi$ and $Y$ by $\varphi$ in (1.4), we get

$$
\xi\left(\alpha_{2}\right)-\alpha_{2}+\lambda \alpha_{1}=0 .
$$

Putting $X=Y=e$ in (1.4), we obtain

$$
2 e\left(\alpha_{1}\right)+2 \lambda^{3}-2 \lambda=0 .
$$

Substitution of $X=e$ and $Y=\varphi e$ in (1.4) yields

$$
e\left(\alpha_{2}\right)+\varphi e\left(\alpha_{1}\right)=0 .
$$

Putting $X=Y=\varphi e$ in (1.4), we infer

$$
2 \varphi e\left(\alpha_{2}\right)-2 \lambda^{3}+2 \lambda=0 .
$$

Since $b=c=0$, the Lie brackets given in (2.4) reduces to

$$
[e, \xi]=e-\lambda \varphi e, \quad[e, \varphi e]=0 \text { and }[\varphi e, \xi]=-\lambda e+\varphi e .
$$

Since $\lambda$ is a positive constant, then from (3.27) and (3.29), we obtain

$$
e\left(\xi\left(\alpha_{1}\right)\right)=e\left(\alpha_{1}\right)-\lambda e\left(\alpha_{2}\right) \text { and } \xi\left(e\left(\alpha_{1}\right)\right)=0 .
$$

Applying the first Lie bracket of (3.32) in the preceding equation, we get $\varphi e\left(\alpha_{1}\right)=e\left(\alpha_{2}\right)$. Hence, equation (3.30) implies $\varphi e\left(\alpha_{1}\right)=e\left(\alpha_{2}\right)=0$. Now, from (3.28), we get $e\left(\xi\left(\alpha_{2}\right)\right)=-\lambda e\left(\alpha_{1}\right)$. Also, we have $\xi\left(e\left(\alpha_{2}\right)\right)=0$. Again, using these two in the first Lie bracket of (3.32) yields $\varphi e\left(\alpha_{2}\right)=e\left(\alpha_{1}\right)$. Applying (3.29) and (3.31) in the preceding relation and using the fact that $\lambda$ is a positive function, we obtain $\lambda=1$. Now, it is easy to check that $\nabla Q=0$. Notice that, a Riemannian 3 -manifold is Ricci parallel if and only if it is locally symmetric. The rest of the proof follows from Theorem 2.6.

As a combination of Proposition 3.2 and Theorem 3.6, we have the following:

Corollary 3.7. If $g$ is a Cotton soliton with potential vector field orthogonal to the Reeb vector field on a 3-dimensional non-unimodular Lie group $M^{3}$ equipped with a left invariant nonKenmotsu almost Kenmotsu structure, then $M^{3}$ is locally isometric to $\mathbb{2}^{2}(-4) \times \mathbb{R}$ and the Cotton soliton is steady.

\section{Example of an almost Kenmotsu 3- $h$-manifold}

Consider $M=\mathbb{R}^{3}$. Let us choose a local orthonormal frame $\left\{e_{1}, e_{2}, e_{3}\right\}$ in such a way that it satisfies the following: 


$$
\left[e_{1}, e_{2}\right]=e_{3}-e_{2}, \quad\left[e_{2}, e_{3}\right]=0 \text { and }\left[e_{3}, e_{1}\right]=-e_{2}+e_{3} .
$$

We define the Riemannian metric $g$ by

$$
g\left(e_{1}, e_{1}\right)=g\left(e_{2}, e_{2}\right)=g\left(e_{3}, e_{3}\right)=1 \text { and } g\left(e_{i}, e_{j}\right)=0 \text { for } i \neq j ; i, j=1,2,3 \text {. }
$$

Consider $e_{1}=\xi$. We define the 1 -form $\eta$ be by $\eta(Z)=g\left(Z, e_{1}\right)$ for any smooth vector field $Z$ on $M$.

Let us define the (1, 1)-tensor fields $\varphi$ and $h$ by

$$
\begin{aligned}
& \varphi\left(e_{1}\right)=0, \quad \varphi\left(e_{2}\right)=e_{3} \text { and } \varphi\left(e_{3}\right)=-e_{2} . \\
& h\left(e_{1}\right)=0, \quad h\left(e_{2}\right)=e_{2} \text { and } h\left(e_{3}\right)=-e_{3} .
\end{aligned}
$$

Using the linearity of $\varphi$ and $g$, we have

$$
\begin{gathered}
\eta\left(e_{1}\right)=1, \\
\varphi^{2}(Z)=-Z+\eta(Z) e_{1} \\
\text { and } g(\varphi Z, \varphi U)=g(Z, U)-\eta(Z) \eta(U)
\end{gathered}
$$

for any smooth vector field $Z, U$ on $M$.

The Levi-Civita connection $\nabla$ of the metric tensor $g$ is given by Koszul's formula:

$$
\begin{aligned}
2 g\left(\nabla_{X} Y, Z\right)= & X g(Y, Z)+Y g(Z, X)-Z g(X, Y) \\
& -g(X,[Y, Z])-g(Y,[X, Z])+g(Z,[X, Y]) .
\end{aligned}
$$

Using the above Koszul's formula, we now calculate the components of the Levi-Civita connection $\nabla$ as follows:

$$
\left(\nabla_{e_{i}} e_{j}\right)=\left(\begin{array}{ccc}
0 & 0 & 0 \\
e_{2}-e_{3} & -e_{1} & e_{1} \\
-e_{2}+e_{3} & e_{1} & -e_{1}
\end{array}\right)
$$

Now, any vector field $X$ on $M$ can be expressed as $X=c_{1} e_{1}+c_{2} e_{2} c_{3} e_{3}$ for some smooth functions $c_{1}, c_{2}$ and $c_{3}$ on $M$. One can easily verify that the relation

$$
\nabla_{X} e_{1}=X-\eta(X) e_{1}-\varphi h X
$$

holds for any smooth vector field $X$ on $M$. Therefore, $(M, \varphi, \xi, \eta, g)$ is an almost Kenmotsu 3-manifold.

Now it can be easily checked that $\left(\nabla_{e_{1}} h\right) X=0$ for any smooth vector field $X$ on $M$. Hence, $M$ is an almost Kenmotsu 3- $h$-manifold.

Here $e_{1}=\xi, e_{2}=e$ and $e_{3}=\varphi e$. Comparing the obtained components of $\nabla_{e_{i}} e_{j}$ with Lemma 2.3, we get $a=b=c=0, \lambda=1, f=2$ and $r=-6$. Then from Lemma 2.4, we can see that $\xi$ is an eigenvector of the Ricci operator $Q$.

Let $V=\alpha e_{2}+\beta e_{3}$, where $\alpha, \beta \in \mathbb{R}$. Then $V$ is orthogonal to $\xi$. Now, the components of $\mathcal{L}_{V} g$ can be obtained as follows:

$$
\begin{gathered}
\left(\mathcal{L}_{V} g\right)\left(e_{1}, e_{1}\right)=0, \quad\left(\mathcal{L}_{V} g\right)\left(e_{2}, e_{2}\right)=0, \quad\left(\mathcal{L}_{V} g\right)\left(e_{3}, e_{3}\right)=0, \\
\left(\mathcal{L}_{V} g\right)\left(e_{1}, e_{2}\right)=-\alpha+\beta, \quad\left(\mathcal{L}_{V} g\right)\left(e_{2}, e_{3}\right)=0 \text { and }\left(\mathcal{L}_{V} g\right)\left(e_{3}, e_{1}\right)=\alpha-\beta .
\end{gathered}
$$

With the help of equation (1.4), one can verify that $g$ is a steady cotton soliton with potential vector field $V=\alpha e_{2}+\alpha e_{3}$ for any real number $\alpha$. 
Also, one can check that $\nabla Q=0$ holds good (see page $5[12]$ ). Then $\nabla R=0$. Hence from Theorem 2.6 , we can say that $M$ is locally isometric to the product space $\mathbb{U}^{2}(-4) \times \mathbb{R}$. This verifies our Theorem 3.6.

\section{References}

1. Blair DE. Contact manifold in riemannian geometry. Lecture notes on Mathematics. Berlin: Springer; 1976. 509.

2. Blair DE. Riemannian Geometry on contact and symplectic manifolds. Progr Math. Boston: Birkhäuser; 2010. 203.

3. Dileo G, Pastore AM. Almost Kenmotsu manifolds and nullity distributions. J Geom. 2009; 93: 46-61.

4. Dileo G, Pastore AM. Almost Kenmotsu manifolds and local symmetry. Bull Belg Math Soc Simon Stevin. 2007; 14: 343-54.

5. Uddin S, Ahsan Z, Yaakub AH. Classification of totally umbilical slant submanifolds of a Kenmotsu manifold. Filomat. 2016; 30(9): 2405-12.

6. Uddin S. Geometry of warped product semi-slant submanifolds of a Kenmotsu manifold. Bull Math Sci. 2018; 8(3): 435-51.

7. Ghosh A, Sharma R. Sasakian manifolds with purely transversal Bach tensor. J Math Phys. 2017; 58: 103502.

8. Kicisel AUO, Sarioğlu O, Tekin B. Cotton flow, class. Quan Grav. 2008; 25: 165019.

9. Chen X. Cotton solitons on almost coKähler 3-manifolds. Quaest Math. doi: 10.2989/16073606. 2020.1767721.

10. Wang W. Almost Kenmotsu 3-h-manifolds with cyclic parallel Ricci tensor. J Nonlinear Sci Appl. 2016; 9: 4206-13.

11. Cho JT, Kimura M. Reeb flow symmetry on almost contact three-manifolds. Differential Geom Appl. 2014; 35: 266-73.

12. Wang Y. Conformally flat almost Kenmotsu 3-manifolds. Med. J. Math. 2017; 14: 186.

13. Wang Y. Three dimensional almost Kenmotsu manifolds with $\eta$-parallel Ricci tensors. J. Kor. Math Soc. 2017; 54: 793-805.

14. Cho JT. Local symmetry on almost Kenmotsu three-manifolds. Hokkaido Math J. 2016; 45: 435-42.

15. Milnor J. Curvature of left invariant metrics on Lie groups. Adv Math. 1976; 21: 293-329.

\section{Corresponding author}

Dibakar Dey can be contacted at: deydibakar3@gmail.com

For instructions on how to order reprints of this article, please visit our website:

www.emeraldgrouppublishing.com/licensing/reprints.htm

Or contact us for further details: permissions@emeraldinsight.com 